\title{
Description of the Diadegma fenestrale (Hymenoptera: Ichneumonidae: Campopleginae) Attacking the Potato Tuber Moth, Phthorimaea operculella (Lep.: Gelechiidae) New to Korea
}

\author{
Jin-Kyung Choi ${ }^{1}$, Ju Il Kim², Min Kwon ${ }^{2}$, Jong-Wook Lee ${ }^{3, *}$ \\ ${ }^{1}$ National Institute of Environmental Research, Incheon 404-708, Korea \\ ${ }^{2}$ Highland Agriculture Research Center, National Institute of Crop Science, RDA, Pyeongchang 232-955, Korea \\ ${ }^{3}$ Department of Life Sciences, Yeungnam University, Gyeongsan 712-749, Korea
}

\begin{abstract}
Diadegma fenestrale is known as a parasitoid of the potato tuber moth, Phthorimaea operculella. The potato tuber moth, Phthorimaea operculella (Zeller) is one of the most destructive pest of potatoes. Also, we found this species attacking the diamondback moth, Plutella xylostella (Lepidoptera: Plutellidae). Ratio of parasitism is $20-30 \%$ and cocoon of lepidopteran was parasitic ichneumonid species after 3 days. This species and the genus Diadegma are recorded for the first time from Korea. In this paper, description of the parasitoid and photographs of the diagnostic characteristics are provided.
\end{abstract}

Keywords: Diadegma fenestrale, Ichneumonidae, parasitoid, Phthorimaea operculella, Korea

\section{INTRODUCTION}

The subfamily Campopleginae, was first described by Förster in 1869 based on the genus Campoplex. Members of this subfamily include 2,133 species in 70 genera from around the world. Among them, 489 species are distributed in the Eastern Palaearctic region, and 23 species have been recorded in Korea (Yu et al., 2005). Yu et al. (2005) listed 23 species belonging to the subfamily Campopleginae from Korea, and Lee and Choi (2007) and Choi and Lee (2008a, 2008b, 2010a, 2010b), and Choi et al. $(2011,2012)$ added 14 species. In this study, we confirmed 38 Korean species in 15 genera.

Most campoplegine species are specialized parasitoids of Lepidopteran or Coleopteran species, and many particularly attack most of the larvae of Lepidoptera as koinobiont endoparasitoids. The taxa of the genus Diadegma includes 121 species with host records. Hosts of this genus include 438 species, and 53 families in five orders, including Coleoptera (phytophagous), Diptera, Hymenoptera (phytophagous, predator, parasitoid), Lepidoptera (phytophagous, predator/flower sucker), and Trichoptera (scavenger). Parasitoids of this genus include 31 species in seven families. The potato tuber moth, Phthorimaea operculella (Zeller) is one of the most destructive pest of potatoes (Rondon, 2010).

In this study, we found a newly recorded species, Diadegma fenestrale (Holmgren, 1860) attacking the potato tuber moth, Phthorimaea operculella (Zeller) in Korea.

The materials used in this study were collected from Jeju, Korea in 2009. The wasps were reared on Phthorimaea operculella in plastic cages under conditions of $25 \pm 2^{\circ} \mathrm{C}, 16 \mathrm{~L}$ : 8D photoperiod, and $50-70 \%$ relative humidity.

Specimens were picked from newly emerging females out of a mass rearing cage and examined by stereo microscopy (Zeiss Stemi SV 11 Apo; Carl Zeiss, Göttingen, Germany). The key characters are shown in photographs that were produced using a Delta imaging system (i-Delta 2.6; iMTechnology, Daejeon, Korea). Abbreviations are as follows: TD, type depository; NR, Naturhistoriska Riksmuseet, Sektionen for Entomologi, S-10405 Stockholm, Sweden; HU, Hokkaido University, Faculty of Agriculture, Entomological Institute, Sapporo, Japan; GUPTA, Entomology \& Nematology Department, University of Florida, Gainesville, FL, USA (V.K. Gupta collection.). (c) This is an Open Access article distributed under the terms of the Creative Commons Attribution Non-Commercial License (http://creativecommons.org/ licenses/by-nc/3.0/) which permits unrestricted non-commercial use, distribution, and reproduction in any medium, provided the original work is properly cited.

pISSN 2234-6953 eISSN 2234-8190
*To whom correspondence should be addressed

Tel: 82-53-810-2376, Fax: 82-53-811-2376

E-mail: jwlee1@ynu.ac.kr 


\section{SYSTEMATIC ACCOUNTS}

Order Hymenoptera

Family Ichneumonidae

Genus Diadegma Förster, 1869

Angitia Holmgren, 1859: 327. Type: Angitia glabricula Holmgren. Monobasic.

Nythobia Förster, 1868: 153. Type: Meloboris pusio Holmgren. New synonymy.

Diadegma Förster, 1868: 153. Type: Campoplex crassicornis Gravenhorst.

Pectinella Morley, 1915: 173. Type: Angitia latungula Thomson. Monobasic.

Areolina Enderlein, 1921: 41. Type: Areolina imbecilla Enderlein.

Nothanomaliudes Viereck, 1925: 272. Type: Nothanomaloides stenosomus Viereck.

Ebiicha Seyrig, 1935: 88. Type: Ebiicha crocata Seyrig. New synonymy.

Neoarthula Rao, 1953: 179. Type: Neoarthula pierisae Rao. Neoangitia Horstmann, 1969: 413. Type: Angitia glabricula Horstmann.

Auma Dbar, 1984: 435. Type: Diadegma elegans Dbar.

Diagnosis. Clypeus moderately convex, its apical margin truncate or weakly convex; lower tooth of mandible equal to upper tooth; genal carina join oral carina; areola longer than wide, areola and petiolar areas usually not separated from transverse carina; tarsal claws pectinate or sometimes pectinate only at the base; discoidella not reaching nervellus; ovipositor more than 2 times as long as apical depth of metasoma but sometimes only as long as apical depth of metasoma, strongly upcurved.

1*Diadegma fenestrale (Holmgren, 1860)

Limneria fenestralis Holmgren, 1860: 1-158. Lectotype: female; TD: NR.

Angitia fenestralis microareolata (Constantineanu \& Petcu, 1960). Type: male.

Angitia fenestralis nigriscapus (Constantineanu \& Petcu, 1960). Type: female.

Diadegma niponica Kusigemati, 1993. Type: female; TD: HU.

Diadegma varuna Gupta, 1974. Type: female; TD: GUPTA.

Material examined. [Lectotype] 1 우

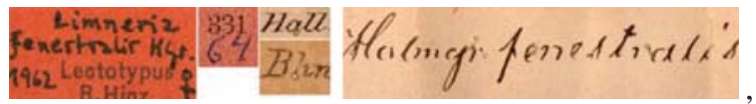

147 지 지 9우 우, Korea: Jeju-do, Seogwipo-si, Daejeong-eup, Sangmo-ri, 28 May 2009, Kim JI, reared from Phthorimaea operculella (Zeller).

Description (female). Body length 5.5-6 mm. Fore wing length 3.5-4 mm. Antenna with 24 flagellomeres.

Color: Head black; mandible brown except apical part black; labrum brown; palpi yellow; scape mostly black, ventral part yellow; antenna black. Mososoma black; tegula pale brown; fore coxa black basally, yellow apically; fore trochanter and trochantellus pale yellow; femur, tibia, and tarsus brown; mid trochanter and trochantellus yellow, femur, tibia, and tarsus brown; hind coxa and trochanter black, trochantellus yellow; femur brown with black spot basally; tibia brown except for pale yellow dorsally with black spots apically and basally; tarsus yellowish brown. Ovipositor brown; ovipositor sheath black; third tergite reddish brown laterally.

Head: Face and frons finely punctate, frons without median vertical ridge; vertex closely and finely punctate more than face; occiput flat, at the edges like the profile of a dish, occipital carina complete; temple finely punctate and weakly convex; clypeus broad and rounded, weakly cancave, not separated from face, clypeal foveae open; mandible short and stout, upper tooth longer than lower; labial palp with four segments, maxillary palp with five segments; inner margins of eyes parallel; ocelli small.

Mesosoma: Pronotum subpolished, upper area with transverse stripe carinae, epomia absent; mesoscutum finely punctate, notauli absent (Fig. 1E); mesopleurum closely punctate and polished, trans-striate; prepectal carina and postpectal carinae complete, sternaulus weakly present basally; speculum convex and shiny, pleural pit strong; scutellum convex, closely punctate, lateral carina absent, postscutellum convex, wide longer than high; metapleurum finely punctate; propodeum with numerous transverse carina, basal area and areola separated by anterior transverse carina, areola and petiolar area incompletely separated, costula complete, petiolar area flat, with transverse carinae (Fig. 1F); spiracle of propodeum a small circle, connected to pleural carina (Fig. 1H); tarsal claw simple (Fig. 1B); fore wing with areolet, second intercubitus opened, basal vein based to nurvulus; second recurrent vein and discocubitus with each other 1 bulla; hind wing with four hamuli, intercubitella with one bulla, nervellus not intercepted, discoidella absent (Fig. 1J).

Metasoma: Slender; petiole with lateral pit; thyridium weak; glymma present; ovipositor upcurved, ovipositor sheath with hairs; middle of seventh sternite cut (Fig. 1I).

Male. Third to fifth tergites reddish brown laterally; thyridium reddish brown; middle of seventh sternite not cut.

Distribution. Korea (new record), Afghanistan, Austria, Azer-

Korean name: ${ }^{1 *}$ 감자뿔나방살이자루맵시벌 (신칭) 

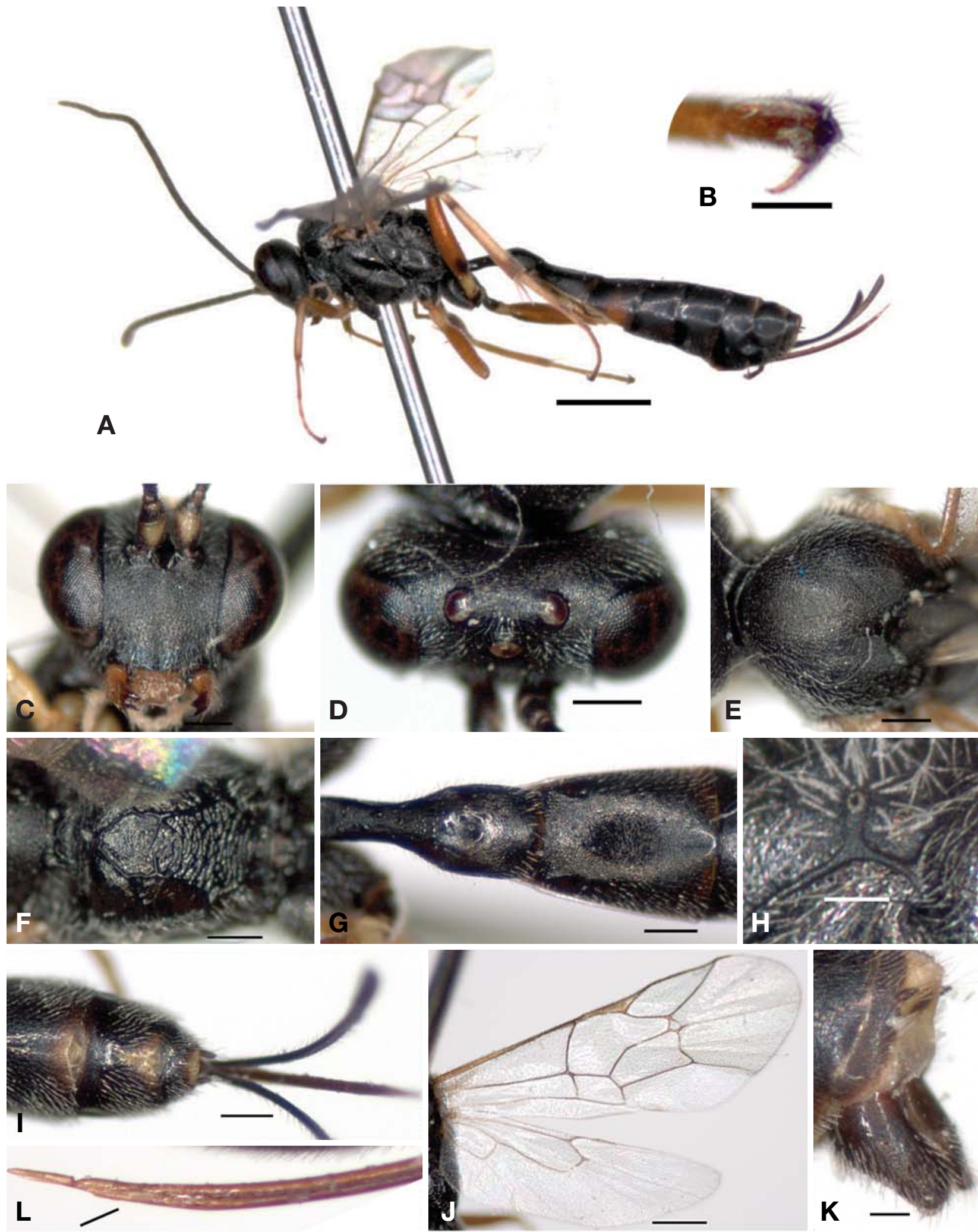

Fig. 1. Diadegma fenestrale (Holmgren, 1860) (A-J, L, female). A, Habitus; $B$, Hind tarsal claw; C, Head in frontal; $D$, Head in dorsal; E, Scutum; F, Propodeum; G, First and second tergites; H, Propodeal spiracle; I, 6-8 tergites; J, Wings; K, Clasper of male; L, Ovipositor. Scale bars: $A=1 \mathrm{~mm}, B, H, K, L=0.1 \mathrm{~mm}, C-G, I=0.2 \mathrm{~mm}, J=0.5 \mathrm{~mm}$ 
baijan, Belgium, Bulgaria, Cyprus, Czech Republic, Czechoslovakia, Denmark, Finland, France, Germany, Hungary, Iceland, India, Ireland, Israel, Italy, Japan, Kazakhstan, Latvia, Lithuania, Moldova, Morocco, Netherlands, New Zealand, Norway, Pakistan, Philippines, Poland, Portugal, Romania, Russia, Spain, Sri Lanka, Sweden, Switzerland, Turkey, Turkmenistan, Ukraine, United Kingdom, and Yugoslavia.

Region. Eastern Palaearctic, Oceanic, Oriental, Western Palaearctic.

Remarks. This species was reared from Phthorimaea operculella (Zeller). Ratio of parasitism is $20-30 \%$. We confirmed parasitic to ichneumonid species after 3 days. Also, we found this species attacking the diamondback moth, Plutella xylostella (Lepidoptera: Plutellidae).

\section{ACKNOWLEDGMENTS}

This study was supported by "The Survey of Korean Indigenous Species" supported by National Institute of Biological Resources (NIBR) of Ministry of Environment of Korea.

\section{REFERENCES}

Choi JK, Lee JW, 2008a. Taxonomic study of the genus Charops Holmgren (Hymenoptera: Ichneumonidae: Campopleginae) from the Easter Palaearctic region. Entomological Research, 38:157-164.

Choi JK, Lee JW, 2008b. Three newly recorded campoplegine species (Hymenoptera: Ichneumonidae: Campopleginae) from Korea. Korean Journal of Systematic Zoology, 24: 285-289.

Choi JK, Lee JW, 2010a. Taxonomic study of the genus Casinaria Holmgren (Hymenoptera: Ichneumonidae: Campopleginae) from Korea. Entomological Research, 40:148-156.

Choi JK, Lee JW, 2010b. Taxonomic study of Korean Eriborus Förster (Hymenoptera: Ichneumonidae: Campopleginae) new to Korea. Entomological Research, 40:236-241.

Choi JK, Jeong JC, Lee JW, 2011. First record of the littleknown genus Hellwigia (Hymenoptera: Ichneumonidae: Campopleginae) from Korea. Korean Journal of Systematic Zoology, 27:170-175.

Choi JK, Jeong JC, Lee JW, 2012. Three species of the subfamily Campopleginae (Hymenoptera: Ichneumonidae) new to Korea. Entomological Research, 42:79-84.

Constantineanu MI, Petcu IP, 1960. Campoplegines (Hym., Ichneumonidae) nouveaux pour la science et pour la faune de la R.P.R. Analele Stiintifice ale Universitatii "Al. I. Cuza" din Iasi. Sect. II a., 6:713-717 (in Rumanian with French \& Russian summaries).

Dbar RS, 1984. A new genus and new species of Ichneumonids of the tribe Limnerini (Hymenoptera, Ichneumonidae) from the USSR and the Mongolian People's Republic. Nasekomye
Mongolii, 9:438-445.

Enderlein G, 1921. Beiträge zur Kenntnis aussereuropäischer Ichneumoniden V. Über die Familie Ophionidae. Stettiner Entomologische Zeitung, 82:3-45.

Förster A, 1868. Monographie der Gattung Campoplex, Grv. Verhandlungen der Zoologisch-Botanischen Gesellschaft in Wien, 18:761-876.

Förster A, 1869. Synopsis der Familien und Gattungen der Ichneumonen. Verhandlungen des Naturhistorischen Vereins der Preussischen Rheinlande und Westfalens, 25(1868):135221.

Gupta VK, 1974. The subfamily name Campopleginae versus Porizontinae (Hymenoptera Ichneumonidae). Entomologist's Gazette, 25:224-226.

Holmgren AE, 1859. Conspectus generum Ophionidum Sueciae. Öfversigt af Kongliga Vetenskaps-Akademiens Förhandlingar, 15:321-330.

Holmgren AE, 1860. Försök till uppställning och beskrifning af de i Sverige funna Ophionider. (Monographia Ophionidum Sueciae). Kungliga Svenska Vetenskapsakademiens Handlingar, 2:1-158.

Horstmann K, 1969. Typenrevision der europäischen Arten der Gattung Diadegma Förster (syn. Angitia Holmgren). Beiträge zur Entomologie, 19:413-472.

Kusigemati K, 1993. Descriptions of two new Ichneumonflies (Hymenoptera) parasitic on diamondback moth, Plutella xylostella L. (Lepidoptera, Yponomeutidae) from Japan. Japanese Journal of Entomology, 61:101-107.

Lee JW, Choi WY, 2007. New species of the genus Skiapus Morley (Hymenoptera: Ichneumonidae: Campopleginae) from Korea. Entomological Research, 37:294-297.

Morley C, 1915. Ichneumonologia Britannica, The Ichneumons of Great Britain. V. Ophioninae. 1914. Brown, London, pp. $1-400$.

Rao SN, 1953. On a collection of Indian Ichneumonidae (Hymenoptera) in the Forest Research Institute, Dehra Dun. Indian Forest Records, 8:159-225.

Rondon SI, 2010. The potato tuberworm: a literature review of its biology, ecology, and control. American Journal of Potato Research, 87:149-166.

Seyrig A, 1935. Mission scientifique de l'Omo. Tome III. Fascicule 18. Hymenoptera, II. Ichneumonidae: Cryptinae, Pimplinae, Tryphoninae et Ophioninae. Memoires du Muséum. National d'Histoire Naturelle, Paris, 4:1-100.

Viereck HL, 1925. A preliminary revision of some Charopsinae, a sub-family of Ichneumonoidea or Ichneumon flies. Proceedings and Transactions of the Royal Society of Canada, 19:259-273.

Yu DS, Van Achterberg C, Horstmann K, 2005. World Ichneumonoidea 2004. Taxonomy, biology, morphology and distribution [Ichneumonidae].-Taxapad 2005 (Scientific names for information management). Interactive Catalogue on CDROM, Vancouver, Canada.

Received July 10, 2012 Revised October 5, 2012 Accepted October 10, 2012 